\title{
ON PRIME ENDS AND LOCAL CONNECTIVITY
}

\author{
LASSE REMPE-GILLEN \\ Dedicated to the memory of Professor Gerald Schmieder
}

\begin{abstract}
Let $U \subset \hat{\mathbb{C}}$ be a simply connected domain whose complement $K=\hat{\mathbb{C}} \backslash U$ contains more than one point. We establish a characterization of the local connectivity of $K$ at a point $z_{0} \in \partial U$ in terms of the prime ends of $U$ whose impressions contain $z_{0}$. Invoking a result of Ursell and Young [UY], we obtain an alternative proof of a theorem of Torhorst, which states that the impression of a prime end of $U$ contains at most two points at which $K$ is locally connected.
\end{abstract}

\section{HistORICAL COMMENT}

This article appeared in the Bulletin of the London Mathematical Society in 2008. In 2012, Donald Sarason kindly pointed out to me that Theorem 1.1 was proved by Marie Torhorst in 1918 in her dissertation at Bonn University. Her original thesis has been lost (a copy exists neither at Bonn nor in her personal Nachlaß), but she published the result in [To]. It seems that this theorem has largely been forgotten: no reference to Torhorst's paper is recorded on MathSciNet, while the latest reference that I was able to find on Zentralblatt dates from 1930. Whyburn [W] references her article in his book Analytic Topology, but does not mention the theory of prime ends (see below).

In the 1960s, Sarason himself wrote a paper [S] with the exact same title as this present note, which also proves Torhorst's result using the more recent work of Ursell and Young. However, his manuscript was not accepted for publication at the time, as he explains:

I submitted the paper to the Michigan Math. J., then edited by George Piranian, the person who taught me about prime ends and much more about complex analysis. (George is one of my mathematical heroes.) George discussed the paper with Collingwood, one of his collaborators. Their conclusion was that interest in prime ends at the time was at such a low ebb that the paper was likely to be largely ignored.

I did publish an abstract of the paper in the Notices of the A.M.S. (Vol 16 (1969), p. 701). At the time the Notices published abstracts of talks given at society meetings, plus what I think were called by-title abstracts, which any member of the society could use to announce a result. If my memory is correct, I received as a result of the abstract only one request for a copy of the paper.

Date October 25, 2018.

2000 Mathematics Subject Classification. Primary 30C35; Secondary 54F15, 30D05, 37F10.

Supported in part by EPSRC grant EP/E017886/1. 
As far as I am aware, Theorem 1.3, which is a characterization of local connectivity at a given point, and of which Theorem 1.1 is a corollary, has not previously appeared elsewhere. (Note, however, that the argument that proves the "only if" direction is the same as the one that appears already in [S], which also contains the "if" direction in the special case that every prime end whose impression contains the point in question is of the first kind.)

Sarason's manuscript [S], along with George Piranian's letter and the announcement in the Notices, are available from my professional website. The preprint version of my original article will follow below, after some additional remarks concerning Marie Torhorst's mathematical work.

\section{FURTHER COMMENTS ON TORHORST'S WORK}

Torhorst's paper [ $\mathrm{To}$ ] contains a number of other interesting results, including the fact that the impression of each prime end can contain at most three points at which the boundary is "accessible from all sides" in the sense of Schoenflies (compare [W, p. 111] for the definition). Moreover, she appears to state and prove for the first time that local connectivity of the boundary of a domain is equivalent to the continuous extension of the Riemann map. This result has come to be extremely prominent, particularly in the area of one-dimensional holomorphic dynamics, but is usually attributed to Carathéodory (compare Corollary 3.2 below). While Carathéodory's theory of prime ends is of course central to the proof, to my knowledge he never made a connection to local connectivity. Indeed, it seems that the latter notion was only developed by Hahn at a time at which Carathéodory's theory was already completed. In view of this fact, it would seem more appropriate to refer to the result as the Carathéodory-Torhorst Theorem.

Today, Torhorst is remembered, if at all, for what is sometimes called the Torhorst Theorem (see [W, Theorem 2.2 in Chapter VI]): the boundary of each complementary domain of a locally connected continuum is itself locally connected. Prof. Walter Purkert has kindly pointed out that Hausdorff also studied this theorem [Ha]. However, this is only a corollary of Torhorst's main results. It is unfortunate that her contributions to the theory of prime ends appear to have been forgotten, particularly considering the relatively small number of female researchers in mathematics at that time.

Torhorst did not become a professional research mathematician - her autobiography articulates a lack of confidence in her own mathematical ability, and in any case, academic research at the time was an almost exclusively male undertaking. (In 1919 Emmy Noether became the first woman to be allowed to to habilitate in mathematics in Germany, with the support of Hilbert and Klein but only after years of considerable resistance from the academic establishment in Göttingen.) A life-long communist, Torhorst was persecuted by the Nazi regime and later became a functionary in the SED, the governing socialist party of East Germany. In 1947, she was appointed Minister of Education in the state of Thüringen, which makes her the first-ever female minister (on state or national level) in the history of Germany. Marie Torhorst died in May 1989 at the age of 100. More information about her life can be found at [Th] and in the sources referenced there. 


\section{INTRODUCTION}

The theory of prime ends was developed by Carathéodory [C] in 1913. One of its central theorems states that the complement $K$ of a simply connected domain $U=\hat{\mathbb{C}} \backslash K$, $\# K>1$, is locally connected if and only if every prime end has trivial impression, which in turn is equivalent to any Riemann map $\varphi: \mathbb{D} \rightarrow U$ having a continuous extension to $\partial \mathbb{D}$. (See Section 3 for a short introduction to the standard definitions and terminology of prime end theory.) This note investigates the question whether there is a relationship between local connectivity of $K$ at a point $z_{0} \in \partial U$ - i.e., the existence of arbitrarily small connected neighborhoods of $z_{0}$ in $K{ }_{1}^{1}$ compare Section 2 - and the structure of the prime ends of $U$ whose impressions contain $z_{0}$.

This question seems very natural, and may be of particular interest due to the prominence that local connectivity of Julia sets and the Mandelbrot set at certain points has received in recent years (see e.g. $[\mathrm{Hu},[\mathrm{K}]$ ). However, it does not appear to have received any treatment in the literature so far.

A naive hope might be that $K$ is locally connected at $z_{0}$ if and only if every prime end impression which contains $z_{0}$ is trivial, but this is false, as the well-known case of the "double comb" shows (Figure 1(a)). However, study of this and similar examples suggests that a nontrivial impression should not contain "too many" points of local connectivity. In this note, we demonstrate that "not too many" can be made very precise. In fact, the example in Figure 1(a) is already best possible.

1.1. Theorem (Prime ends and local connectivity).

Let $U \subset \hat{\mathbb{C}}$ be a simply connected domain such that $K:=\hat{\mathbb{C}} \backslash U$ contains more than one point, and let $p$ be a prime end of $U$. Then the impression $I(p)$ contains at most two points at which $K$ is locally connected.

Remark. We also show that, if furthermore the prime end $p$ is symmetric (see Section 4), then $I(p)$ contains at most one point at which $K$ is locally connected.

The proof of Theorem 1.1 uses a result (Theorem 4.4) concerning the "wings" (aka the left and right cluster sets) of a prime end that was proved by Ursell and Young [UY] in 1951 and deserves to be far better known.

We will deduce Theorem 1.1 from Theorem 4.4 by developing a necessary and sufficient criterion for local connectivity at $z_{0}$ in terms of the prime ends of $U$. To state this result, we introduce the following notion.

1.2. Definition (Strong minimality).

Let $p$ be a prime end, and let $z_{0}$ belong to the impression of $p$. We say that $z_{0}$ is strongly minimal in $p$ if, for every sequence $w_{j} \in U$ converging to $p$ which does not accumulate on $z_{0}$, there is a curve $\Gamma:[0, \infty) \rightarrow U$ that converges to $p$ and passes through all $w_{j}$ but does not accumulate on $z_{0}($ as $t \rightarrow \infty)$.

This terminology is motivated by such a point being minimal with respect to Ursell and Young's ordering by priority; see Definition 4.2 .

\footnotetext{
${ }^{1}$ Sometimes this property is instead referred to as connected im kleinen.
} 
1.3. Theorem (Characterization of local connectivity). Let $z_{0} \in \partial U$. Then $K$ is locally connected at $z_{0}$ if and only if $z_{0}$ is strongly minimal in every prime end whose impression contains $z_{0}$.

The proof of Theorem 1.3 is elementary, and the result might almost be considered a restatement of the definition of local connectivity. However, it does provide an interesting and quite satisfying answer to our initial question about the connection between prime ends and local connectivity; in particular it contains Carathéodory's characterization of local connectivity of $K$ (see Corollary 3.2). Theorem 1.1 follows from Theorem 1.3 and the aforementioned result by Ursell and Young (compare Corollary 4.5).

Basic notation. We denote the complex plane by $\mathbb{C}$, the Riemann sphere by $\hat{\mathbb{C}}$, and the unit disk by $\mathbb{D}$. We write $\mathbb{D}_{\delta}(z)$ for the (Euclidean) disk of radius $\delta$ around $z$.

Organization of the article. In Section 2, we define local connectivity at a point and discuss a number of variations of this definition. We also develop a simple characterization of local connectivity of $K$ at $z_{0}$. Section 3 provides a short review of the theory of prime ends and the proof of Theorem 1.3. In Section 4, we discuss Theorem 4.4, by Ursell and Young, and deduce Theorem 1.1 from it. For completeness, we provide a proof of Theorem 4.4 in the Appendix.

Acknowledgments. I had many interesting and enjoyable discussions on this subject over the years, in particular with Chris Bishop, David Epstein, Christian Pommerenke, Lex Oversteegen, Dierk Schleicher and the late Gerald Schmieder. I would like to thank Walter Bergweiler for a choice of seminar topic that not only introduced me to Pommerenke's excellent book [P], but also led me to discover Theorem 1.1] as an undergraduate at Kiel University in 1999. Finally, I am grateful to Christian Pommerenke and Lex Oversteegen for encouraging me to publish this note.

\section{LOCAL CONNECTIVITY}

For the remainder of the paper, let $U \subset \hat{\mathbb{C}}$ be a simply connected domain whose complement $K:=\hat{\mathbb{C}} \backslash U$ contains at least two points. We will assume without loss of generality that $\infty \in U$, so $K$ is a compact, connected subset of the complex plane.

Recall that $K$ is called locally connected if every point $z \in K$ has arbitrarily small connected (relative) neighborhoods in $K$. Following Milnor [M, Chapter 17], we say that $K$ is locally connected at a point $z_{0} \in K$ if $z_{0}$ has arbitrarily small connected neighborhoods in $K$. Sometimes this property is instead referred to as "connected im kleinen", and the term "locally connected at $z_{0}$ " is instead reserved for what Milnor calls "openly locally connected", see below.

Observe that local connectivity of $K$ at $z_{0}$ is equivalent to the condition that the connected component of $\left\{z \in K:\left|z-z_{0}\right| \leq \delta\right\}$ containing $z_{0}$ is a neighborhood of $z_{0}$ in $K$ for all $\delta>0$.

Remarks on the definition of local connectivity at a point. We note that there are many equivalent definitions of local connectivity of the entire space $K$ which result in different concepts when considered only near a point $z_{0}$. For example, we might say that $K$ is openly locally connected at $z_{0}$ if the point has arbitrarily small open neighborhoods 


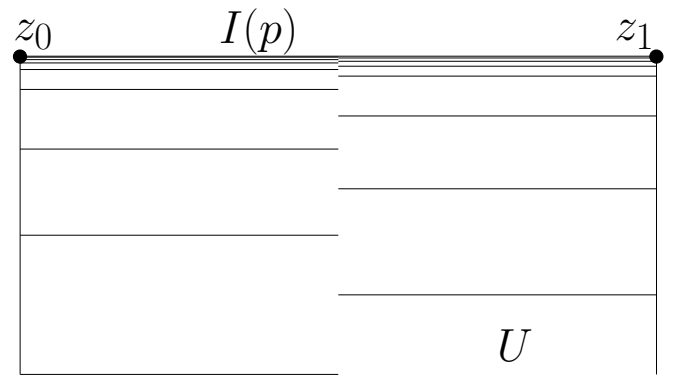

(a) The double comb

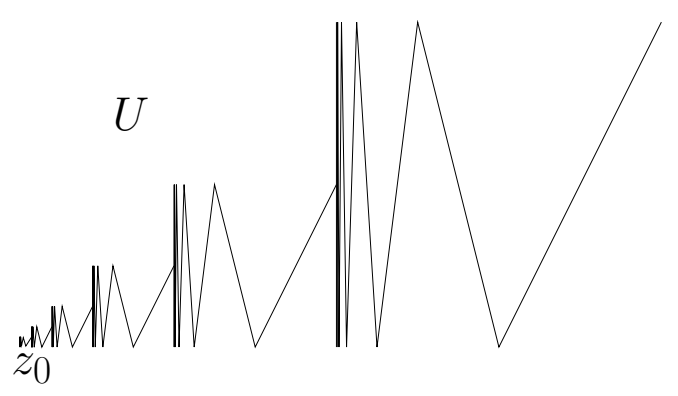

(c) Path connectivity

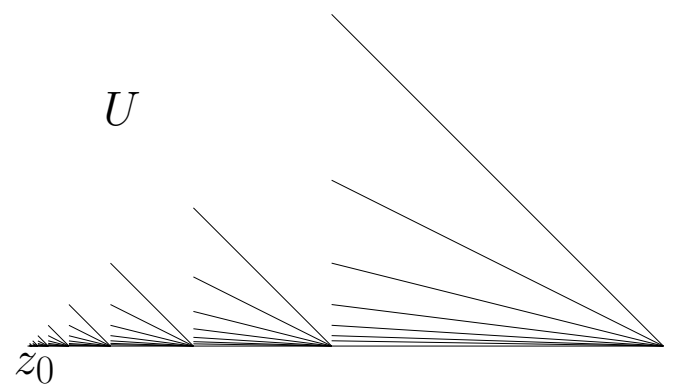

(b) The witch's broom

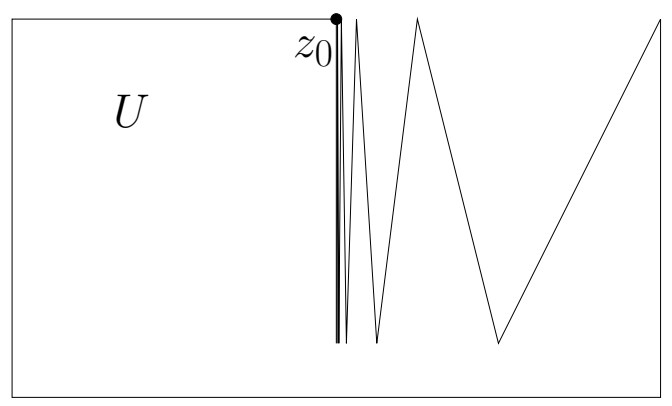

(d) $K$ vs. $\partial U$

Figure 1. Several examples of simply connected domains and their boundaries. (a) illustrates Theorem 1.1 the interval at the top of the figure is the impression of a single prime end $p$, and $K=\hat{\mathbb{C}} \backslash U$ is locally connected at the two endpoints $z_{0}$ and $z_{1}$. Also note that these endpoints are strongly minimal in the sense of Definition 1.2, while the interior points are not. Examples (b) to (d) illustrate our remarks on the definition of local connectivity in Section 2 .

in $K$. It is well-known that $K$ is locally connected if and only if it is openly locally connected at every point. However, if $K$ is not locally connected as a whole, then it is quite possible that there are points $z_{0} \in K$ where the set is locally connected but not openly locally connected. (A famous example is the "witch's broom"; see Figure 1(b).)

Similarly, local connectivity of $K$ implies that $K$ is locally arc-connected, but it is clearly possible for a compact space $K$ to be locally connected at a point $z_{0}$, but not to contain any nontrivial curves passing through $z_{0}$. (See Figure 1(c).)

Finally, Carathéodory's theorem is often phrased as a statement on local connectivity of the boundary $\partial U=\partial K$, rather than of $K$. Indeed, these two are equivalent when we consider the entire space; however, local connectivity of $\partial U$ at $z_{0}$ is a strictly stronger condition than local connectivity of $K$ at $z_{0}$. (See Figure $1(\mathrm{~d})$.)

We believe that, in our context, local connectivity of $K$ at $z_{0}$ is the most natural among the possible concepts to consider. This is vindicated by the fact that we are able to obtain natural characterizations of this notion. Also, we should point out that our choice places the fewest restrictions on the point $z_{0}$, so that Theorem 1.1 takes its strongest form with this definition. 
Separation theorems and preliminaries. We say that two points $z, w \in \hat{\mathbb{C}}$ are separated by a set $K$ if they belong to different components of $\hat{\mathbb{C}} \backslash K$. Similarly, if $U \subset \hat{\mathbb{C}}$ is a domain, we sometimes say that $z$ and $w$ are separated by $K$ in $U$ if they belong to different components of $U \backslash K$.

We repeatedly use the following standard separation theorem [Ne, p. 110] due to Janiszewski: if $K_{1}$ and $K_{2}$ are compact subsets of the sphere whose intersection is connected, then a pair of points which is not separated by either of $K_{1}$ and $K_{2}$ is also not separated by the union $K_{1} \cup K_{2}$.

We will also invoke the boundary bumping theorem [Na, Theorem 5.6]: if $E$ is a subset of a compact, connected metric space $K$, then the boundary of every connected component of $E$ intersects the boundary of $E$ (in $K$ ).

Let us furthermore remind the reader that a crosscut $C$ of a simply connected domain $U \subset \hat{\mathbb{C}}$ is a closed Jordan arc which intersects $\hat{\mathbb{C}} \backslash U$ exactly in its two endpoints. Every crosscut separates $U$ into precisely two components. (Since $U$ is homeomorphic to the complex plane, this is an immediate consequence of the Jordan Curve Theorem; compare [P, Proposition 2.12]. Observe that the argument applies more generally to any injective curve in $U$ which accumulates at $\partial U$ in both directions; we use this fact in the Appendix.)

Finally, we note the following simple result.

2.1. Lemma (Curves in a subdomain).

Let $V \subset U$ be a domain, and let $z_{0} \in \partial U$. Suppose that $\operatorname{dist}\left(z_{0}, \partial V \cap U\right)>\varepsilon$.

If $w_{1}, w_{2} \in V$ can be connected by a curve $\gamma \subset U$ with $\operatorname{dist}\left(\gamma, z_{0}\right)>\varepsilon$, then such a curve also exists in $V$.

Proof. Let us set $A:=\hat{\mathbb{C}} \backslash V \supset K$ and $B:=K \cup \overline{\mathbb{D}_{\varepsilon}\left(z_{0}\right)}$. By assumption, neither $A$ nor $B$ separate $w_{1}$ and $w_{2}$. We claim that $X:=A \cap B$ is connected.

Indeed, we have $X=K \cup\left((U \backslash V) \cap \overline{\mathbb{D}_{\varepsilon}\left(z_{0}\right)}\right)$. Suppose, by contradiction, that there was a component $L$ of $X$ other than the one containing $K$; then in particular $z_{0} \notin L$. Pick some boundary point $w$ of $L$ relative to $\overline{\mathbb{D}_{\varepsilon}\left(z_{0}\right)}$. Then $w \in \partial V$, but because $w \in L \subset U$ and $\left|w-z_{0}\right| \leq \varepsilon$, this contradicts our assumptions.

So $X$ is connected. By Janiszewski's theorem, $A \cup B=(\mathbb{C} \backslash V) \cup \overline{\mathbb{D}_{\varepsilon}\left(z_{0}\right)}$ does not separate $w_{1}$ and $w_{2}$, as desired.

A characterization of local connectivity. Our proof of Theorem 1.3 (and, by extension, of Theorem 1.1) relies on the following necessary and sufficient condition for local connectivity of $K$ at $z_{0}$. Compare Figure 2(c).

2.2. Proposition (Characterization of local connectivity).

Let $z_{0} \in \partial U$. Then $K$ is locally connected at $z_{0}$ if and only if the following holds: for every $\delta>0$, there is $\varepsilon>0$ such that every point $w \in U \backslash \mathbb{D}_{\delta}\left(z_{0}\right)$ can be connected to $\infty$ by a curve $\gamma \subset U \backslash \overline{\mathbb{D}_{\varepsilon}\left(z_{0}\right)}$.

Proof. Suppose that $K$ is locally connected at $z_{0}$, and let $\delta>0$. Let $L$ be the connected component of $K \cap \overline{\mathbb{D}_{\delta}\left(z_{0}\right)}$ containing $z_{0}$. Then $L$ is a compact, connected neighborhood 
of $z_{0}$ in $K$. I.e., there is $\varepsilon>0$ such that

$$
\overline{\mathbb{D}_{\varepsilon}\left(z_{0}\right)} \cap K \subset L .
$$

Let $w_{1}, w_{2} \in U \backslash \mathbb{D}_{\delta}\left(z_{0}\right)$. Applying Janiszewski's theorem to $K$ and $L \cup \overline{\mathbb{D}_{\varepsilon}\left(z_{0}\right)}$, we see that $K \cup \overline{\mathbb{D}_{\varepsilon}\left(z_{0}\right)}$ does not separate $w_{1}$ from $w_{2}$, as claimed.

For the converse direction, suppose that $K$ is not locally connected at $z_{0}$. Then there is $\delta>0$ such that the connected component $L$ of $A:=K \cap \overline{\mathbb{D}_{\delta}\left(z_{0}\right)}$ with $z_{0} \in L$ is not a neighborhood of $z_{0}$ in $K$.

Let $\varepsilon>0$. Then there is a point $z \in A \backslash L$ with $\left|z-z_{0}\right| \leq \varepsilon$. Write $A=A_{0} \cup A_{1}$, where $A_{0}$ and $A_{1}$ are disjoint compact sets with $L \subset A_{0}$ and $z \in A_{1}$. By the boundary bumping theorem, both $L$ and the component of $A$ containing $z$ intersect $\partial \mathbb{D}_{\delta}\left(z_{0}\right)$; in particular, they both intersect the circle $\partial \mathbb{D}_{\varepsilon}\left(z_{0}\right)$.

So we can pick an arc $C$ of $\partial \mathbb{D}_{\varepsilon}\left(z_{0}\right) \backslash A$ which has one endpoint in $A_{0}$ and the other in $A_{1}$. Then $\bar{C}$ is a crosscut of $U$; let $V$ be the component of $U \backslash C$ which does not contain $\infty$. We claim that $V$ contains some point $w$ with $\left|z_{0}-w\right| \geq \delta$. Indeed, applying Janiszewski's theorem first to the sets $A_{0}$ and $\bar{C}$ and then to $A_{0} \cup \bar{C}$ and $A_{1}$, we see that we can connect $\infty$ to any point $\tilde{w} \in V$ by a curve not intersecting $A \cup C$. If $\left|\tilde{w}-z_{0}\right|>\delta$, we set $w:=\tilde{w}$. Otherwise let $w$ be the last intersection point of this curve with $\partial \mathbb{D}_{\delta}\left(z_{0}\right)$; then $w \in V$.

By definition of $V$, any curve $\gamma$ connecting $\infty$ to $w$ must intersect $C$, and hence have $\operatorname{dist}\left(\gamma, z_{0}\right) \leq \varepsilon$, as required.

\section{PRIME ENDS}

We refer the reader to [M, Chapter 17] for an excellent short treatment of the theory of prime ends, and to [P. Chapter 2] for further results. Here, we will only introduce the basic definitions, and give no proofs. As before, $U$ is a simply connected domain whose complement $K$ omits more than one point, and $\infty \in U$ for simplicity.

If $C$ is a crosscut of $U$ with $\infty \notin C$, then $U \backslash C$ has exactly one component which does not contain $\infty$; let us denote this component by $U_{C}$. A prime end of $U$ is represented by a sequence of pairwise disjoint crosscuts $\left(C_{n}\right)$ which satisfy $\operatorname{diam} C_{n} \rightarrow 0$ and $C_{n+1} \subset \overline{U_{C_{n}}}$. Two such sequences $\left(C_{n}\right)$ and $\left(\tilde{C}_{n}\right)$ represent the same prime end if $\tilde{C}_{j} \subset U_{C_{n}}$ for all $n$ and all sufficiently large $j$, and vice versa.

The impression of $p$ is defined as

$$
I(p):=\bigcap_{n} \overline{U_{C_{n}}} \subset \partial U
$$

an impression is trivial if it consists of a single point. The set of principal points, $\Pi(p) \subset I(p)$, consists of those points which are accumulated on by some sequence of crosscuts representing $p$.

There is a natural way to define a topology on

$$
\breve{U}:=U \cup\{p: p \text { is a prime end of } U\}
$$

such that a sequence $w_{j} \in U$ converges to $p$ if and only if $w_{j} \in U_{C_{n}}$ for all $n$ and all sufficiently large $j$. With this topology, $\breve{U}$ is homeomorphic to the closed unit disk $\overline{\mathbb{D}}$. 


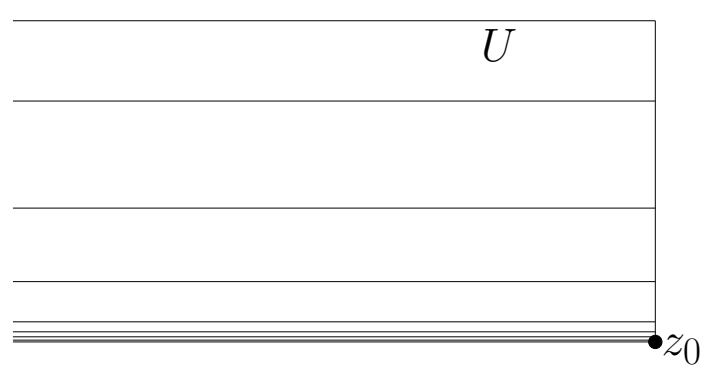

(a) The single comb

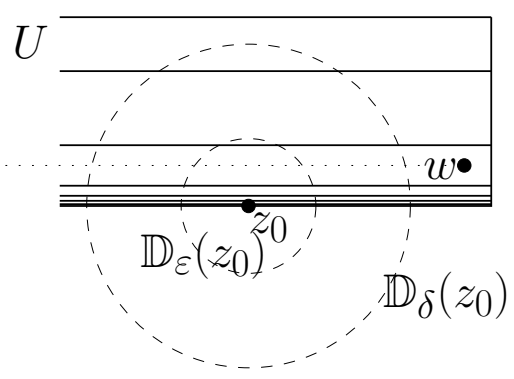

(c) Proposition 2.2

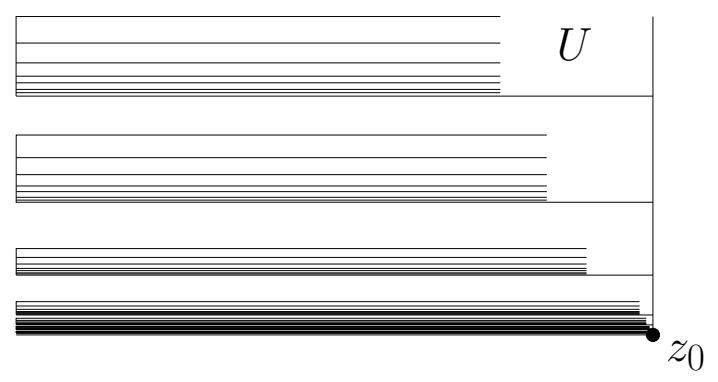

(b) A modified comb

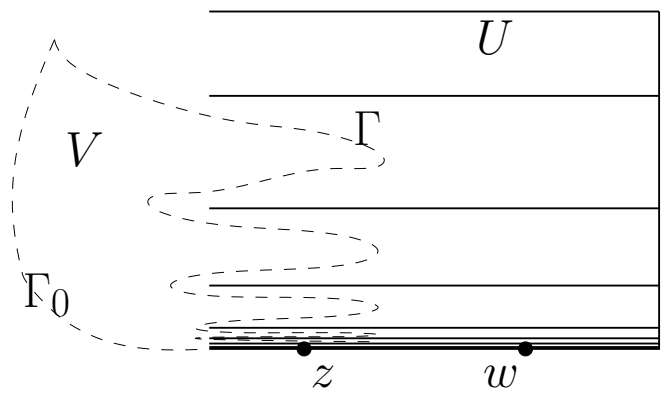

(d) Proof of Theorem 4.4

Figure 2. The single comb and variations. In (a), the set $K=\hat{\mathbb{C}} \backslash U$ is locally connected at $z_{0}$ (but not in any other point of the interval at the bottom edge of the picture). In contrast, the point $z_{0}$ is not a point of local connectivity in (b): this shows that the structure of a prime end's impression, and the order of priority from Definition 4.2, is not sufficient to detect local connectivity. Figure (c) illustrates the statement of Proposition 2.2 ; $K$ is not locally connected at $z_{0}$, and any curve connecting $w$ to infinity must intersect the disk $\mathbb{D}_{\varepsilon}\left(z_{0}\right)$. Finally, (d) indicates the setup in the proof of Theorem 4.4; the curve $\Gamma$ accumulates on $z$ but not on $w$, while $\Gamma_{0}$ accumulates on the set $\Pi(p)$ of principal points. Together with their accumulation sets, they separate $w$ from the region $V$.

In fact, if $\varphi: \mathbb{D} \rightarrow U$ is a conformal isomorphism, then $\varphi$ extends continuously to a homeomorphism $\varphi: \overline{\mathbb{D}} \rightarrow \breve{U}$.

We say that a curve $\Gamma:[0, \infty) \rightarrow U$ converges to $p$ if $\Gamma(t) \rightarrow p$ in the topology of $\breve{U}$. Note that the set of accumulation points of such a curve $\Gamma$ necessarily contains $\Pi(p)$.

Proof of Theorem 1.3. Suppose that $K$ is locally connected at $z_{0}$, and consider a prime end $p$ with $z_{0} \in I(p)$. Let $\left(w_{j}\right)$ be a sequence as in the definition of strong minimality (Definition 1.2), and set $\delta:=\inf \left|z_{0}-w_{j}\right|$. By Proposition 2.2, there is $\varepsilon_{0}>0$ such that each $w_{j}$ can be connected to $\infty$ by a curve $\gamma_{j}$ with $\operatorname{dist}\left(\gamma_{j}, z_{0}\right)>\varepsilon_{0}$.

Let $\left(C_{n}\right)$ be a sequence of crosscuts representing $p$, and set $U_{n}:=U_{C_{n}}$. Without loss of generality, we may assume that no $C_{n}$ has $z_{0}$ as an endpoint (otherwise, we simply remove this crosscut from the sequence). 
For sufficiently large $j$, we have $w_{j} \in U_{n}$, and hence $\gamma_{j} \cap C_{n} \neq \emptyset$. Since $\operatorname{dist}\left(\gamma_{j}, z_{0}\right)>\varepsilon_{0}$ and $\operatorname{diam}\left(C_{n}\right) \rightarrow 0$, we see that

$$
\varepsilon_{1}:=\inf _{n} \operatorname{dist}\left(z_{0}, C_{n}\right)>0
$$

we set $\varepsilon:=\min \left(\varepsilon_{0}, \varepsilon_{1}\right)$.

By construction, we can connect $w_{j}$ and $w_{j+1}$ by a curve $\Gamma_{j}$ which does not intersect $\overline{\mathbb{D}_{\varepsilon}\left(z_{0}\right)}$ (e.g., $\Gamma_{j}=\gamma_{j} \cup \gamma_{j+1}$ ). If $w_{j}$ and $w_{j+1}$ both belong to the same $U_{n}$, we can, by Lemma 2.1, furthermore choose $\Gamma_{j}$ such that $\Gamma_{j} \subset U_{n}$. The curve $\Gamma:=\bigcup \Gamma_{j}$ converges to the prime end $p$, contains all points $w_{j}$, and does not intersect $\overline{\mathbb{D}_{\varepsilon}\left(z_{0}\right)}$.

Now suppose, conversely, that $K$ is not locally connected at $z_{0}$. Then, by Proposition 2.2, there is a constant $\delta>0$ with the following property: for every $n \in \mathbb{N}$ there is a point $\omega_{n} \in U$ with $\left|\omega_{n}-z_{0}\right| \geq \delta$ such that any curve connecting $\omega_{n}$ to $\infty$ within $U$ must pass within distance at most $1 / n$ of $z_{0}$.

Clearly we have $\omega_{n} \rightarrow \partial U$. Let $p$ be some accumulation point of $\omega_{n}$ in the prime end compactification $\breve{U}$ of $U$. We define a sequence $\left(w_{j}\right)_{j \geq 0}$ by setting $w_{0}:=\infty$ and $w_{j}:=\omega_{n_{j}}$, where $\left(\omega_{n_{j}}\right)_{j \geq 1}$ is a subsequence converging to $p$ in $\breve{U}$.

Then any curve $\Gamma$ tending to $p$ and containing all points $w_{j}$ will, in particular, connect $w_{0}=\infty$ to $w_{j}=\omega_{n_{j}}$. Hence $\operatorname{dist}\left(\Gamma, z_{0}\right)=0$ by choice of $\left(\omega_{n}\right)$, which implies that $z_{0} \in I(p)$ and that $z_{0}$ is not strongly minimal in $p$.

3.1. Corollary (Local connectivity at principal points).

Suppose that $K$ is locally connected at a principal point $z_{0}$ of $p$. Then $I(p)=\left\{z_{0}\right\}$.

Proof. We prove the contrapositive. Suppose that $I(p) \neq\left\{z_{0}\right\}$; then we can pick some $z \in I(p) \backslash\left\{z_{0}\right\}$. Take a sequence of points $w_{k} \in U$ converging to $z$ such that $w_{j}$ converges to $p$ in the prime end topology. Since $z_{0}$ is a principal point, any curve converging to $p$ (containing all $w_{j}$ or not) must accumulate on $z_{0}$, and hence $z_{0}$ is not strongly minimal. By Theorem 1.3, $K$ is not locally connected at $z_{0}$.

Remark. This fact is easy to prove also without Theorem 1.3. Let $\left(C_{n}\right)$ be a sequence of crosscuts converging to $z_{0}$. Pick a closed connected neighborhood $Z_{\varepsilon}$ of $z_{0}$ in $K$ satisfying $Z_{\varepsilon} \subset \mathbb{D}_{\varepsilon}\left(z_{0}\right)$; we may suppose without loss of generality that $\hat{\mathbb{C}} \backslash Z_{\varepsilon}$ is connected. Then, for sufficiently large $n$, the curve $C_{n}$ is a crosscut of $\hat{\mathbb{C}} \backslash Z_{\varepsilon}$. It follows that $U_{n}$, and hence $I(p)$, is contained in $\overline{\mathbb{D}_{\varepsilon}\left(z_{0}\right)}$. Since $\varepsilon$ was arbitrary, the claim follows.

3.2. Corollary (Carathéodory's theorem).

The set $K$ is locally connected if and only if every prime end impression is trivial.

Proof. Since $K$ is locally connected at every point of its interior, we only need to consider local connectivity at points of $\partial U$.

If $K$ is not locally connected at some point $z_{0} \in \partial U$, then by Theorem 1.3 there is some prime end $p$ with $z_{0} \in I(p)$ such that $z_{0}$ is not strongly minimal in $p$. In particular, there is some sequence $\left(w_{j}\right)$ converging to $p$ but not converging to $z_{0}$, so $I(p)$ is not trivial.

On the other hand, if there is some nontrivial prime end impression $I(p)$, then $K$ is not locally connected at any point of $\Pi(p)$ by the previous corollary. 


\section{The LEFT AND RIGHT WINGS OF A PRIME END}

The article [UY] studied the left and right wings of a prime end. Today these are more commonly referred to as the left and right cluster sets, or as the one-sided impressions CP2. We prefer to use "wing" here, as it seems to be the original term used when these sets are investigated as topological objects related to the domain $U$ and its boundary, rather than as an aspect of a conformal mapping $\varphi: \mathbb{D} \rightarrow U$. For simplicity, we will nonetheless not give a purely topological definition, but rather use the conformal mapping $\varphi$.

Let $p$ be a prime end of $U$. We say that a curve $\Gamma:[0, \infty) \rightarrow U$ converges to $p$ from the left if $\Gamma$ converges to $p$, and furthermore

$$
\operatorname{Im}\left(\frac{\varphi^{-1}(\Gamma(t))}{p}\right) \geq 0
$$

for all sufficiently large $t$. (Here we again identify the prime end $p$ with the corresponding point $p=e^{2 \pi i \vartheta}$ on the unit circle.) We say that any accumulation point $z_{0} \in I(p)$ of such a curve $\Gamma$ belongs to the left wing of $p$, and write $I^{+}(p)$ for all such points.

The right wing $I^{-}(p)$ is defined analogously. We note that $I(p)=I^{+}(p) \cup I^{-}(p)$ and $\Pi(p) \subset I^{-}(p) \cap I^{+}(p)$.

\subsection{Example.}

In Figure 1(a), the interval at the top of the picture is the only nontrivial prime end impression. The midpoint $m$ of this interval is the unique principal point; the left and right wings are the intervals $\left[z_{0}, m\right]$ and $\left[m, z_{1}\right]$, respectively.

In Figures 2(a) and 2(b), the prime end at the bottom of the picture has a trivial right wing, containing only the unique principal point, while the left wing consists of the entire interval at the bottom of the picture.

The prime end $p$ is called symmetric if $I^{-}(p)=I^{+}(p)$. By the Collingwood Symmetry Theorem $[\mathrm{P}$, Proposition 2.21], all but countably many prime ends are symmetric. Compare [CP1] for interesting results on symmetric prime ends (among other things).

4.2. Definition (Priority).

Let $z, w \in I^{-}(p)$. We say that $z$ has priority over $w\left(\right.$ in $\left.I^{-}(p)\right)$ if every curve $\Gamma$ which converges to $p$ from the left and accumulates on $w$ must also accumulate on $z$.

(Priority in $I^{+}(p)$ is defined analogously.)

\subsection{Example.}

In the left wing of the nontrivial prime end expression of Figure 2(a), the order of priority coincides with horizontal order: if $z, w$ belong to the interval at the bottom of the picture and $z$ is to the left of $w$, then $z$ has priority over $w$. The same is true in Figure $2(b)$.

4.4. Theorem (Priority is a total relation $[\mathrm{UY}]$ ).

Let $z, w$ belong to the same wing of the prime end $p$. Then $z$ has priority over $w$ or $w$ has priority over $z$. 
Now suppose that $z_{0} \in I(p)$ is strongly minimal in $p$. Then $z_{0}$ is minimal with respect to priority; indeed, $z_{0}$ cannot have priority over any point in either wing. Hence Theorem 4.4 implies:

4.5. Corollary (At most two strongly minimal points). Each wing of the prime end $p$ contains at most one point which is strongly minimal in $p$.

Proof of Theorem 1.1. We just proved that $I(p)$ contains at most two points which are strongly minimal (at most one for each wing). We also know by Theorem 1.3 that local connectivity of $K$ at $z_{0}$ requires strong minimality of $z_{0}$ in $p$. This proves the theorem.

If the prime end is symmetric, then both wings are equal and Corollary 4.5 implies that $I(p)$ contains at most one strongly minimal point, establishing the remark after the statement of Theorem 1.1.

Remark. One might ask whether strong minimality can be expressed solely in terms of the order of priority on $I(p)$; this is not the case. Indeed, the first two examples in Figure 2 both have a prime end whose impression is the bottom interval of the picture, and as discussed above the corresponding orders of priority coincide. However, in the first figure $z_{0}$ is strongly minimal, while in the second figure it is not.

\section{Appendix: Proof of the Ursell-Young Theorem}

Proof of Theorem 4.4. Suppose that $z$ and $w$ both belong to (say) the left wing of the prime end $p$, and that $w$ does not have priority over $z$. That is, there is a curve $\Gamma$ : $[0, \infty) \rightarrow U$ converging to $p$ from the left whose accumulation set $A$ contains $z$ but not $w$. For simplicity, let us assume that $\Gamma$ is injective. (It is not hard to see that we can always find an injective curve with the same accumulation set. Alternatively, with minor modifications the proof will also apply in the general case.) We need to show that $z$ has priority over $w$. We may assume that $z \notin \Pi(p)$, as every principal point has priority over all other points by definition.

Let $\Gamma_{0}:[0, \infty) \rightarrow U$ be the "central curve"

$$
\Gamma_{0}(t):=\varphi\left(e^{-1 / t} \cdot p\right)
$$

separating the left and right wings of $p$. The limit set of $\Gamma_{0}$ is the set of radial limit points of $\varphi$ at $p$, which is well-known to consist exactly of $\Pi(p)[\mathrm{P}$, Theorem 2.16]. We may assume without loss of generality that $\Gamma(0)=\Gamma_{0}(0)$ and that $\Gamma((0, \infty)) \cap \Gamma_{0}=\emptyset$ (recall that $\Gamma$ converges to $p$ from the left).

Since $\Gamma_{0}$ and $\Gamma_{1}$ accumulate only on $\partial U$, the set $U \backslash\left(\Gamma_{0} \cup \Gamma\right)$ has exactly two components. Since $\Gamma_{0}$ and $\Gamma$ both converge to the same prime end $p$, one of these components, call it $V$, accumulates on no other prime ends in the topology of $\breve{U}$. (Compare Figure 2(d).) We pick some arbitrary base point $x \in V$.

Claim 1. We have $\partial V=\Gamma_{0} \cup \Gamma \cup A=: F$. In particular, $F$ separates $x$ from $w$.

Proof. Let $\tilde{U}$ be the component of $\hat{\mathbb{C}} \backslash A$ containing $U$ and let $\tilde{V}$ be the component of $\tilde{U} \backslash\left(\Gamma_{0} \cup \Gamma\right)$ containing $V$. Then $V=\tilde{V} \cap U$; we need to show that $V=\tilde{V}$. 
Indeed, otherwise $\tilde{V}$ is a neighborhood of some point of $\partial U$; in particular $\tilde{V}$ and hence $V$ contains some crosscut of $U$. However, this is a contradiction to the choice of $V$, since every crosscut accumulates on two distinct prime ends of $U$.

Claim 2. Let $\varepsilon$ be sufficiently small. Let $T>0$ be minimal with $|\Gamma(T)-z| \leq \varepsilon$. Then the set

$$
K_{1}(\varepsilon):=\overline{\mathbb{D}_{\varepsilon}(z)} \cup \Gamma([T, \infty)) \cup A
$$

does not separate $x$ and $w$ (in $\hat{\mathbb{C}}$ ).

Proof. Let $w^{\prime} \in U$ with $\left|w-w^{\prime}\right|<\operatorname{dist}(w, A)$. By connecting $x$ to $w^{\prime}$ in $U$ and $w^{\prime}$ to $w$ by a straight line segment, we obtain a curve $\alpha \subset \mathbb{C} \backslash A$ which connects $x$ to $w$. Set $\delta:=\operatorname{dist}(\alpha, A)$. If $\varepsilon$ is sufficiently small, then $K_{1}(\varepsilon)$ is contained in a $\delta$-neighborhood of $A$, and hence does not intersect $\alpha$.

Let $\left(C_{n}\right)$ be a sequence of crosscuts representing $p$; we may choose these so that $C_{n} \cap \Gamma_{0}$ consists of a single point for every $n$. Let $U_{n}$ be the component of $U \backslash\left(C_{n} \cup \Gamma_{0}\right)$ which contains $\Gamma(t)$ for large $t$. Then a curve converges to $p$ from the left if and only if it is eventually contained in every $U_{n}$.

Claim 3. Let $\varepsilon>0$. Then there are $n_{0}$ and $\delta$ with the following property. If $n \geq n_{0}$ and $w^{\prime} \in U_{n}$ with $\left|w-w^{\prime}\right|<\delta$, then any curve in $U_{n}$ connecting $w^{\prime}$ to $C_{n}$ intersects $\overline{\mathbb{D}_{\varepsilon}(z)}$.

Proof. By decreasing $\varepsilon$, if necessary, we may assume that $x$ can be connected to every crosscut $C_{n}$ by a curve $\beta_{n} \subset V$ which does not intersect $\overline{\mathbb{D}_{\varepsilon}(z)}$. This is possible because $\Gamma_{0}$ does not accumulate on $z$.

By Claim 2, we may also assume that $K_{1}=K_{1}(\varepsilon)$ does not separate $x$ and $w$. Consider the set

$$
K_{2}:=\overline{\mathbb{D}_{\varepsilon}(z)} \cup A \cup \Gamma_{0} \cup \Gamma([0, T]) .
$$

Observe that $K_{1} \cap K_{2}=\mathbb{D}_{\varepsilon}(z) \cup A$ and $F \subset K_{1} \cup K_{2}$. Hence it follows from Claim 1 and Janiszewski's theorem that $K_{2}$ separates $x$ and $w$.

Choose $\delta$ sufficiently small that $\mathbb{D}_{\delta}(w) \cap K_{2}=\emptyset$, and choose $n_{0}$ such that $C_{n} \cap \overline{\mathbb{D}_{\varepsilon}(z)}=\emptyset$ and $U_{n} \cap \Gamma([0, T])=\emptyset$ for $n \geq n_{0}$.

If $w^{\prime} \in U_{n}$ with $n \geq n_{0}$ and $\left|w^{\prime}-w\right|<\delta$, then $K_{2}$ separates $w^{\prime}$ and $x$. Let $\gamma \subset U_{n}$ be a curve connecting $w^{\prime}$ to $C_{n}$. Combining $\gamma$ with a piece of $C_{n}$ and the curve $\beta_{n}$, we obtain a curve in $U \backslash\left(\Gamma_{0} \cup \Gamma([0, T])\right)$ connecting $w^{\prime}$ to $x$. This curve must intersect $K_{2}$, and hence $\overline{\mathbb{D}_{\varepsilon}(z)}$. Since $C_{n}$ and $\beta_{n}$ do not intersect this disk, it follows that $\gamma$ intersects $\overline{\mathbb{D}_{\varepsilon}(z)}$, as claimed.

The proof of Theorem 4.4 is now complete: suppose that $\tilde{\Gamma}$ is a curve converging to $p$ from the left and accumulating on $w$, and let $\varepsilon>0$. Let $\delta$ and $n_{0}$ be as in Claim 3, and pick $n \geq n_{0}$ sufficiently large so that $\tilde{\Gamma} \not \subset U_{n}$. Since $\tilde{\Gamma}$ contains some point $w^{\prime} \in U_{n}$ with $\left|w^{\prime}-w\right|<\delta$, it follows that $\tilde{\Gamma}$ intersects $\overline{\mathbb{D}_{\varepsilon}(z)}$.

\section{REFERENCES}

[C] Constantin Carathéodory, Über die Begrenzung einfach zusammenhängender Gebiete, Math. Ann. 73 (1913), 323-370.

[CP1] J. J. Carmona and C. Pommerenke, On prime ends and plane continua, J. London Math. Soc. (2) 66 (2002), no. 3, 641-650. 
[CP2] Joan J. Carmona and Christian Pommerenke, Decomposition of continua and prime ends, Comput. Methods Funct. Theory 3 (2003), no. 1-2, 253-272.

[Ha] Felix Hausdorff, Beweis des Satzes von M. Torhorst, in: Gesammelte Werke, Volume III: "Deskriptive Mengenlehre und Topologie", Springer 2008, S. 832-834.

[Hu] J. H. Hubbard, Local connectivity of Julia sets and bifurcation loci: three theorems of J.-C. Yoccoz, Topological methods in modern mathematics (Stony Brook, NY, 1991), Publish or Perish, Houston, TX, 1993, pp. 467-511.

$[\mathrm{K}] \quad$ Jan Kiwi, Real laminations and the topological dynamics of complex polynomials, Adv. Math. 184 (2004), no. 2, 207-267.

[M] John Milnor, Dynamics in one complex variable, third ed., Annals of Mathematics Studies, vol. 160, Princeton University Press, Princeton, NJ, 2006.

[Na] Sam B. Nadler, Jr., Continuum theory. An introduction, Monographs and Textbooks in Pure and Applied Mathematics, vol. 158, Marcel Dekker Inc., New York, 1992.

[Ne] M. H. A. Newman, Elements of the topology of plane sets of points, Cambridge, At the University Press, 1951, 2nd ed.

[P] Christian Pommerenke, Boundary behaviour of conformal maps, Grundlehren der Mathematischen Wissenschaften, vol. 299, Springer-Verlag, Berlin, 1992.

[S] Donald Sarason, On prime ends and local connectivity, Manuscript, ca. 1968.

[Th] Michael Wolf Thomas, ZeitLebensZeiten: Marie Torhorst, accessed on 2014-02-18 at http://www.zeitlebenszeiten.de/html/marie_torhorst.html and archived by WebCite at http://www.webcitation.org/6NTx4ZApX.

[To] Marie Torhorst, Über den Rand der einfach zusammenhängenden ebenen Gebiete, Math. Z. 9 (1921), no. 1-2, 44-65.

[UY] H. D. Ursell and L. C. Young, Remarks on the theory of prime ends, Mem. Amer. Math. Soc., 3 (1951), 29.

[W] Gordon Thomas Whyburn, Analytic Topology, American Mathematical Society Colloquium Publications, v. 28, American Mathematical Society, New York, 1942. MR 0007095 (4,86b)

Department of Mathematical Sciences, University of Liverpool, Liverpool, United KINGDOM L69 7ZL

E-mail address: 1.rempe@liverpool.ac.uk 